\title{
Circular RNA expression profiling identifies specific circular RNAs in tongue squamous cell carcinoma
}

\author{
TAI WEI ${ }^{1}$, PENG YE $^{2}$, GUANG-YAN YU ${ }^{3}$ and ZU-YAN ZHANG ${ }^{1}$ \\ ${ }^{1}$ First Clinical Division, Peking University Hospital of Stomatology, Beijing 100034; ${ }^{2}$ Department of Stomatology, \\ Beijing Hospital, National Centre of Gerontology, Beijing 100730; ${ }^{3}$ Department of Oral and Maxillofacial Surgery, \\ Peking University School and Hospital of Stomatology, Beijing 100181, P.R. China
}

Received June 18, 2019; Accepted November 6, 2019

DOI: $10.3892 / \mathrm{mmr} .2020 .10980$

\begin{abstract}
Tongue squamous cell carcinoma (TSCC) is the most frequent type of oral cancer associated with high malignancy. Circular RNAs (circRNAs) are a form of non-coding RNA with stable and conserved expression in mammalian cells. The aim of the present study was to investigate circRNAs expression profiles in TSCC, and examine the roles and potential mechanisms of circRNA-081069 (circ_081069). A high-throughput circRNA microarray analysis of tumor samples and adjacent normal tissues from four patients with TSCC was performed. Bioinformatic analysis was conducted to screen the differentially expressed circRNAs. Reverse transcription-quantitative PCR was performed to confirm the microarray results. A migration assay and proliferation assay were performed to detect the migratory and proliferative ability of TSCC cells. A luciferase assay was conducted to investigate the interaction between circ_081069 and microRNA (miRNA/miR)-665. In total, 335 circRNAs were found to be differentially expressed in tumor tissues. Among them, 59 were upregulated and 276 were downregulated $(\mathrm{P}<0.05$; fold change $\geq 2$ or $\leq 0.5$ ). A total of seven circRNAs, including two upregulated and five downregulated circRNAs, were further confirmed using quantitative PCR analysis in the ten paired TSCC tissues and adjacent normal tissues. The present study showed that circRNA_081069 promoted the migratory and proliferative ability of TSCC cells in vitro. Furthermore, the potential circRNA-miRNA interactions were predicted, and the present results identified miR-665 as a miRNA target of circ_081069. The present results suggested that circRNAs may be involved in TSCC development, and understanding the
\end{abstract}

Correspondence to: Professor Zu-Yan Zhang, First Clinical Division, Peking University Hospital of Stomatology, 37A Xishiku Street, Beijing 100034, P.R. China

E-mail: zhangzy-bj@vip.sina.com

Contributed equally

Key words: tongue squamous cell carcinoma, circular RNAs, microarray, circular RNA_081069 interaction between circ_081069 and miR-665 may facilitate the development of novel diagnostic and therapeutic targets for TSCC.

\section{Introduction}

Tongue squamous cell carcinoma (TSCC) is the most common type of oral squamous cell carcinoma comprising $41 \%$ of all cases (1), and its frequency is increasing (2). The 5 year survival rate of TSCC is $30-50 \%$, and it is influenced by the high rate of proliferation and early lymph node metastasis (2). Current standard treatments for TSCC include surgical resection combined with postoperative radiotherapy and chemotherapy (2). Although advances have been made in recent years, the identification of a treatment remains a challenge for TSCC. A large proportion of patients with TSCC suffer from postoperative oral defects and lymph node metastasis in multiple regions (3). The lack of effective targets to control and monitor the disease is one of the principal problems in TSCC treatment (3). Therefore, it would be beneficial to identify novel biological targets to control tumors effectively and reduce the impairment of oral functions.

Circular RNAs (circRNAs) are a type of non-coding RNAs, which form covalently closed continuous loops with the 3'- and 5'- ends joined (4). Due to the distinctive molecular structure, circRNAs are resistant to the degradation from RNAses, resulting in a stable and conserved expression in the cytoplasm (5). circRNAs were thought to be the results of defective RNA splicing events and have been only recently identified (6). A number of previous studies have reported that circRNAs play important roles in carcinogenesis and cancer development by acting as microRNA (miRNA) sponges, regulating gene expression at the transcriptional and translational levels $(6,7)$. The circular transcript ciRS-7 was found to bind miR-7, resulting in the inhibition of miR-7 (8). In addition, miR-7 served as an epidermal growth factor receptor (EGFR) suppressor by binding to the EGFR mRNA 3'-untranslated region (8). The overexpression of ciRS-7 was observed to increase the expression levels of EGFR and activate its downstream signaling pathway through inhibiting miR-7 expression (7). circRNAs also regulate cancer cell biological features by altering the genomic DNA (9). Therefore, identifying the functions of circRNAs may improve 
the understanding of the molecular mechanisms underlying cancer development and may provide potential targets for cancer treatment. Notably, the expression patterns and potential roles of circRNAs in TSCC remain poorly understood.

In the present study, the expression profile of various circRNAs was investigated in TSCC and was compared with the expression profile of circRNAs in paired adjacent normal tissues using microarray analysis. The identification of the differentially expressed circRNAs in TSCC may provide novel insights into the diagnosis and treatment of TSCC.

\section{Materials and methods}

Patients. The study was approved by The Ethics Committee of Peking University School and Hospital of Stomatology (app roval no. PKUSSIRB-2013009). The patient consents were acquired before tissue collection.

The present study included 14 patients (males, 8; females, 6; mean age, 50.7 years; age range, 39-73 years) who underwent surgery at Peking University School and Hospital of Stomatology to pathologically confirm primary TSCC, between January 2017 and December 2017. None of the patients had received any anti-cancer therapy before sample collection.

Part of the resected tumor tissues and paired adjacent normal tissues ( $\geq 2 \mathrm{~cm}$ from the tumor margins) were collected. Of the tissues collected, four pairs of tumor and adjacent normal tissues were used for microarray analysis and ten pairs of tumor and adjacent normal tissues were used for validation. The tissue pairs selected for each analysis occurred randomly based on the patient inclusion criteria. Tumor clinical stage and histological grading were classified based on the 8th edition of the TNM classification of the Union for International Cancer Control (10).

Histological evaluation. Paraffin embedded consecutive tissue sections $(5 \mu \mathrm{m})$ were fixed in $4 \%$ paraformaldehyde at room temperature for $24 \mathrm{~h}$ and subsequently stained with $0.5 \%$ hematoxylin solution for $30 \mathrm{~min}$ at room temperature and $0.5 \%$ eosin solution for $10 \mathrm{~min}$ at room temperature. The sections were observed and photographed using an Olympus CKX41 light microscope (magnification x10; Olympus Corporation).

RNA extraction. Total RNA from the ten pairs of tissue samples was isolated using TRIzol ${ }^{\circledR}$ reagent (Thermo Fisher Scientific, Inc.) according to the manufacturer's instruction. RNA quantity and quality were assessed using the NanoDrop ND-100 spectrometer (Thermo Fisher Scientific, Inc.), and RNA integrity was tested by agarose gel electrophoresis.

Labeling and hybridization and microarray analysis. Sample labeling and array hybridization were performed based on the manufacturer's instruction (Arraystar, Inc.). The acquired hybridized array data were analyzed by Agilent Feature Extraction software (version 11.0.1.1; https://www.agilent. com/en/product/mirna-microarray-platform/mirna-microarraysoftware/feature-extraction-software-228496/download-softwarefeature-extraction-software) (11). Quantile normalization of raw data and subsequent data processing were conducted using R software limma package (version 3.1.2; http://www. bioconductor.org/packages/release/bioc/html/limma.html). When comparing two groups (TSCC vs. paracancer), the fold change between the groups for each circRNA was calculated. The statistical significance of the difference was estimated by paired Student's t-test. circRNAs with fold changes $\geq 2$ or $\leq 0.5$ and $\mathrm{P}<0.05$ were selected as differentially expressed circRNAs. CircRNA/miRNA interactions were predicted using the TargetScan (release 7.2; http://www.targetscan. org) and miRanda (release 2010; http://www.microrna.org) databases.

Quantitative (q)PCR. cDNA samples were prepared from total RNA of TSCC samples using the PrimeScript ${ }^{\mathrm{TM}}$ RT reagent kit (Takara Bio, Inc.), according to the manufacturer's protocol. The following conditions were used for the reverse transcription: $37^{\circ} \mathrm{C}$ for $15 \mathrm{~min}$ and $85^{\circ} \mathrm{C}$ for $10 \mathrm{sec}$. In total, two upregulated circRNAs and five downregulated circRNAs were detected on a PikoReal Real-Time PCR System (Thermo Fisher Scientific, Inc.) using the SYBR ${ }^{\circledR}$ Green qPCR kit (Thermo Fisher Scientific, Inc.). The primer sequences were listed in Table I. The following thermocycling conditions were used for the qPCR: Initial denaturation at $95^{\circ} \mathrm{C}$ for $10 \mathrm{~min} ; 35$ cycles of $95^{\circ} \mathrm{C}$ for $10 \mathrm{sec}$ and $60^{\circ} \mathrm{C}$ for $40 \mathrm{sec}$; and a final extension at $72^{\circ} \mathrm{C}$ for $10 \mathrm{~min}$. The relative expression of circRNAs was calculated using the $2^{-\Delta \Delta \mathrm{Cq}}$ method (12), $\beta$-actin was used as the internal reference gene to normalize the expression data.

Cell culture and transfection. Human TSCC cell line, SCC15 and SCC25, were purchased from the American Type Culture Collection. Cells were cultured in DMEM/F12 medium (Gibco; Thermo Fisher Scientific, Inc.) with 10\% FBS (Gibco; Thermo Fisher Scientific, Inc.) at $37^{\circ} \mathrm{C}$ with $5 \% \mathrm{CO}_{2}$ for 3 days before use.

Silencing RNA (siRNA) against circ_081069 (sicirc_081069; cat. no. siBDM2500) and siRNA control (cat. no. siBDM2500) were purchased from Guangzhou RiboBio Co., Ltd. miR-665 mimic (ACCAGGAGGCUGAGGCCCCU; cat. no. 4464066) and negative control (cat. no. 4464066) were purchased from Thermo Fisher Scientific, Inc. and $100 \mathrm{nM}$ of each was transfected into TSCC cells using Lipofectamine ${ }^{\circledR}$ 3000 reagent (Thermo Fisher Scientific, Inc.), according to the manufacturer's protocol. Cells were collected for future experiments following $24 \mathrm{~h}$ of transfection.

Migration assay. Cell migration was measured by Transwell assay. A total of $1 \times 10^{4}$ SCC15 and SCC25 cells were seeded in a 24-well cell culture chamber (pore size, $8 \mu \mathrm{m}$; Corning, Inc.) in $200 \mu 1$ serum-free DMEM/F12 medium. The lower chamber was plated in DMEM/F12 medium supplemented with $20 \%$ FBS. After $24 \mathrm{~h}$ incubation at $37^{\circ} \mathrm{C}$ and $5 \% \mathrm{CO}_{2}$, cells on the lower side of the membrane were fixed in $95 \%$ ethanol at room temperature for $10 \mathrm{~min}$ and stained with $0.5 \%$ hematoxylin at room temperature for $30 \mathrm{~min}$ followed by $0.5 \%$ eosin staining at room temperature for $30 \mathrm{~min}$. The whole membrane was photographed using an Olympus CKX41 light microscope (magnification x20; Olympus Corporation) and the cells on the membrane were quantified using ImageJ version 1.44 software (National Institutes of Health), as previously described (13). All experiments were performed in triplicate and cells transfected with control siRNA acted as the control group. 
Table I. circRNA primers for quantitative PCR analysis.

\begin{tabular}{|c|c|c|}
\hline Gene & Primer sequence $\left(5^{\prime} \rightarrow 3^{\prime}\right)$ & Product size (base pairs) \\
\hline \multirow[t]{2}{*}{$\beta$-actin (Human) } & F: GTGGCCGAGGACTTTGATTG & 73 \\
\hline & R: CCTGTAACAACGCATCTCATATT & \\
\hline \multirow[t]{2}{*}{ hsa_circRNA_000780 } & F: TAGGAAACCTGCTGTGGAGTG-3 & 108 \\
\hline & R: AAGGGAACTATACAAGGAAATGC & \\
\hline \multirow[t]{2}{*}{ hsa_circRNA_102039 } & F: CTATCATTCACAAAGGGAAAACTAC & 164 \\
\hline & R: CCATAACTGGAGTAACCGCTG & \\
\hline \multirow[t]{2}{*}{ hsa_circRNA_003251 } & F: GGAGAAGACGACGACCCACTA & 113 \\
\hline & R: TAGGACAGGGCCTTCTTTGAC & \\
\hline \multirow[t]{2}{*}{ hsa_circRNA_045179 } & F: GCTGCTGTGCAAGAAACGG & 139 \\
\hline & R: CACCTGGCTGAACTTCTGTGACT & \\
\hline \multirow[t]{2}{*}{ hsa_circRNA_081069 } & F: CTGGACTTCCTGGCTTCAAA & 164 \\
\hline & R: TCCTCTATCTCCGGCTGGG & \\
\hline \multirow[t]{2}{*}{ hsa_circRNA_087212 } & F: TTCAATACCATCCTTACCACC & 113 \\
\hline & R: CTCTGATTTCTTTGTTAGTTCTTG & \\
\hline \multirow[t]{2}{*}{ hsa_circRNA_000317 } & F: AAAGGGCCAGAGGTAGACAT & 79 \\
\hline & R: GCAAATCAAAGTCAGGCATAG & \\
\hline
\end{tabular}

F, forward; R, reverse; circRNA, circular RNA.

Proliferation assay. Proliferation of SCC15 and SCC25 cells were evaluated by a Cell Counting Kit-8 (CCK8) cell viability assay (Dojindo Molecular Technologies, Inc.), according to the manufacturer's protocol. For the CCK- 8 assay, $5 \times 10^{3}$ cells were seeded into 96 -well plates. Following incubation at $37^{\circ} \mathrm{C}$ for 48 and $72 \mathrm{~h}$, cell viability was measured at a wavelength of $450 \mathrm{~nm}$ using a spectrophotometric plate reader.

Flow cytometry. For cell apoptosis analysis, $1 \times 10^{4}$ cells were seeded into 6-well plates. After transfection for $48 \mathrm{~h}$, cells were resuspended with $100 \mu \mathrm{l}$ binding buffer after centrifugation at $200 \mathrm{x} \mathrm{g}$ at room temperature for $5 \mathrm{~min}$. Annexin V-FITC (20 $\mu \mathrm{g} / \mathrm{ml}$; Dojindo Molecular Technologies, Inc.) and propidium $(50 \mu \mathrm{g} / \mathrm{ml}$; Dojindo Molecular Technologies, Inc.) were added to cells and incubated for $15 \mathrm{~min}$ at room temperature. The cell apoptotic rate was measured using a BD FACSCalibur ${ }^{\mathrm{TM}}$ flow cytometer (BD Biosciences) and analyzed with CellQuest $^{\mathrm{TM}}$ version 7.5.3 software (BD Biosciences).

Dual-luciferase reporter assay. The sequences containing the binding site of miR-665 in circRNA_081069 (416-435 bp) and the mutant sequences (TCCTGG>CGGCCA) were synthesized by Shanghai Generay Biotech Co., Ltd. and inserted into the luciferase reporter vector psiCHECK-2 (Promega Corporation). Both plasmids were transfected into cells using Lipofectamine ${ }^{\circledR} 3000$ reagent (Thermo Fisher Scientific, Inc.), according to the manufacturer's protocol. Luciferase activity was measured using the Dual-Luciferase Reporter Assay kit (Promega Corporation), according to the manufacturer's instructions following $24 \mathrm{~h}$ of transfection. Relative luciferase activity was normalized to Renilla luciferase activity.

Statistical analysis. Data are presented as the mean \pm SEM. The statistical significance of microarray data was analyzed by fold change using a paired Student's t-test and the false discovery rate was calculated to correct the P-value using SPSS Statistics version 20.0 software (IBM Corp.). Fold change $\geq 2.0$ or $\leq 0.5$ and $\mathrm{P}<0.05$ were applied to find the differentially expressed circRNAs. The remaining statistical analysis was performed using SPSS Statistics version 20.0 software (IBM Corp.). Differences were determined by a paired Student's t-test for paracancer and TSCC groups. $\mathrm{P}<0.05$ was considered to indicate a statistically significant difference.

\section{Results}

Patient characteristics. The clinical characteristics of the 14 patients with pathologically confirmed primary TSCC are presented in Table II. Tissue samples of four patients were used for microarray analysis and the remaining ten pairs of tissue specimens were used for further validation. In total, eight patients with TSCC were male and six patients were female. Moreover, eight patients were classified as T1 and T2 stage, and six patients were classified as T3 and T4 stage. Lymph node metastasis was present in ten patients. Histopathologic characteristics were assessed by hematoxylin \& eosin staining (Fig. 1A-C).

Analysis of differentially expressed circRNAs. Arraystar human circRNA microarray was used to screen 11,916 circRNAs, and differentially expressed circRNAs were identified between TSCC and paracancer tissues. Hierarchical clustering was performed to group circRNAs according to the expression levels among samples (Fig. 1D). The threshold was set as fold change $\geq 2.0$ or $\leq 0.5$ and $\mathrm{P}<0.05$ (Fig. $1 \mathrm{E}$ and $\mathrm{F}$ ). The results showed that 335 circRNAs were differentially expressed, including 59 upregulated circRNAs and 276 downregulated circRNAs (Fig. 1G). The top 15 upregulated and 
Table II. Clinical characteristics of the 14 patients with tongue squamous cell carcinoma.

\begin{tabular}{ccllll}
\hline Case no. & Age (years) & Sex & Histologic differentiation & TNM stage & Admission date \\
\hline 1 & 40 & Male & Moderately & T4aN1M0 & $26 / 04 / 2017$ \\
2 & 44 & Male & Well-moderately & T2N2M0 & $30 / 05 / 2017$ \\
3 & 53 & Male & Poorly & T4aN2M0 & $17 / 06 / 2017$ \\
4 & 49 & Male & Moderately & T1N0M0 & $17 / 06 / 2017$ \\
5 & 64 & Male & Well-moderately & T4aN1M0 & $30 / 06 / 2017$ \\
6 & 39 & Male & Moderately & T4aN1M0 & $30 / 06 / 2017$ \\
7 & 40 & Female & Well-moderately & T2N1M0 & $07 / 07 / 2017$ \\
8 & 55 & Female & Well-moderately & T2N0M0 & $13 / 07 / 2017$ \\
9 & 47 & Male & Well-moderately & T1N0M0 & $13 / 07 / 2017$ \\
10 & 62 & Female & Well-moderately & T1N1M0 & $13 / 07 / 2017$ \\
11 & 73 & Female & Poorly & T4aN2M0 & $31 / 08 / 2017$ \\
12 & 49 & Female & Moderately & T1N2M0 & $11 / 12 / 2017$ \\
13 & 55 & Male & Moderately & T2N0M0 & $15 / 02 / 2017$ \\
14 & 40 & Female & Well-moderately & T4aN1M0 & $20 / 01 / 2017$ \\
\hline
\end{tabular}

$\mathrm{T}$, tumour; $\mathrm{N}$, node; $\mathrm{M}$, metastasis.

top 15 downregulated circRNAs, combined with the detailed molecular information, are presented in Tables III and IV. Among the upregulated circRNAs, there were 50 exonic, seven intronic, one intergenic and one sense overlapping. Among the downregulated circRNAs, there were 251 exonic, 11 intronic, 11 sense overlapping, two antisense and one intergenic (Fig. 1H).

$q P C R$ validation. To validate the microarray results, two upregulated circRNAs (circ_045179 and circ_081069) and five downregulated circRNAs (circ_000317, circ_087212, circ_000780, circ_102039 and circ_003251) were selected from the differentially expressed circRNAs in the microarray. In total, ten pairs of TSCC tissues and paracancer tissues were examined by qPCR to confirm the expression levels of the selected circRNAs. The expression patterns of selected circRNAs showed the same consistency with the microarray results (Fig. 2). Of them, circ_081069 and circ_087212 were the most upregulated and downregulated circRNA, respectively (Fig. 2).

circRNA and miRNA interaction analysis. Previous studies have reported that circRNAs mainly function as miRNA sponges and serve important roles in gene regulation $(6,7)$. The circRNA/miRNA interaction has been shown to be involved in cancer progression, and could be exploited as a potential therapeutic target. To analyze the potential functions of the selected circRNAs, circRNA/miRNA interactions were predicted using TargetScan (release 7.2; http://www.targetscan.org/) and miRanda (release 2010; http://www.microrna.org). A total of two circRNAs (circ_081069 and circ_087212) were examined in detail using the circRNA/miRNA interaction analysis (Fig. 3). The potential miRNA targets of circ_081069 included miR-4733-3p, miR-665 and miR-412-3p. The potential miRNAs interacting with circ_087212 included miR-29a-5p, miR-6873-3p, miR-6809-3p, miR-6515-3p and miR-5584-3p.
A previous study showed that miR-665 suppresses osteosarcoma cell proliferation and invasion by inhibiting the small GTPase protein Rab23 (14). In addition, miR-29a regulates the expression levels of p21, p53 and survivin in cancer cells, resulting in enhanced cell proliferation (15). However, the function of the remaining miRNAs on cancer development remains unclear.

miR-665 is a target of circ_081069. In the present study, the most upregulated circRNA in the microarray analysis, circ_081069, was selected to investigate its potential biological role in TSCC and identify its potential miRNA targets. After knockdown of circ_081069 in TSCC cells by siRNA (Fig. 4A), the migratory and proliferative ability were suppressed significantly (Fig. 4B-D); however, apoptosis was not affected (Fig. S1). The present results suggested that circ_081069 may exert oncogenic effects by regulating cell migration and proliferation. Using bioinformatic prediction methods circ_081069 was predicted to interact with miR-665, possibly acting as a competing endogenous RNA. Using qPCR, the present study found that miR-665 levels were decreased in four TSCC tissues (Fig. 4E). In order to further investigate the direct interaction between circ_081069 and miR-665, a luciferase reporter assay was performed. The present results showed that overexpression of miR-665 inhibited the wild-type circ_081069 reporter, whereas no significant difference was found between the cells overexpressing miR-665 and the negative control in the mutant circ_081069 reporter (Fig. 4F and G). These results suggested that circ_081069 promoted TSCC cell migration and proliferation. Furthermore, miR-665 was able to interact with circ_081069, in line with the bioinformatic prediction results.

\section{Discussion}

In the present study, microarray analysis of differentially expressed circRNAs was conducted by comparing the 
A

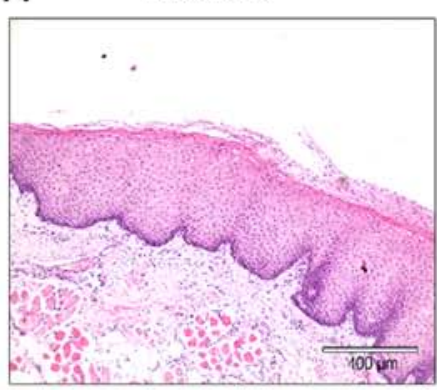

B

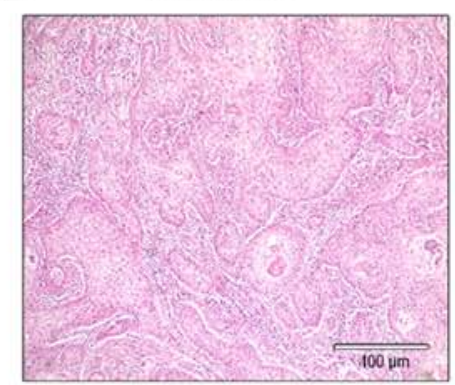

C

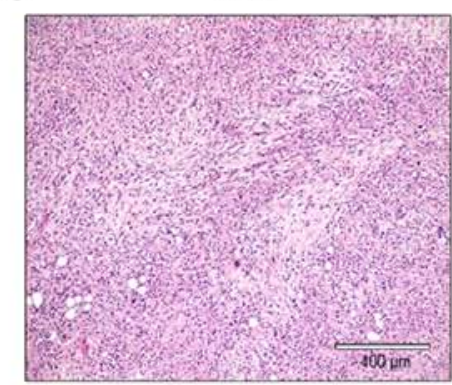

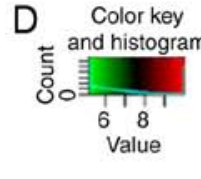
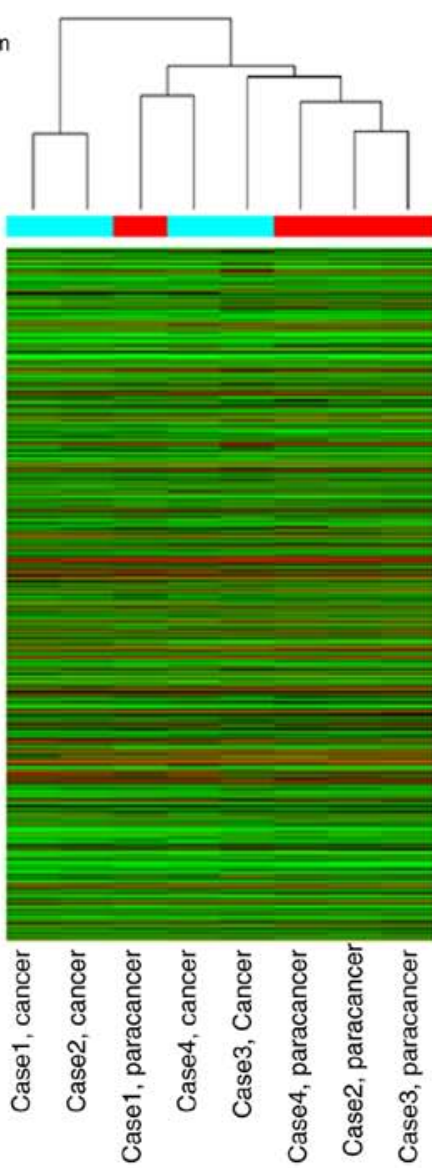

G

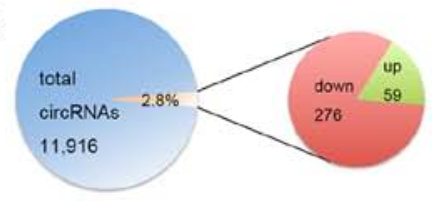

$\mathrm{H}$

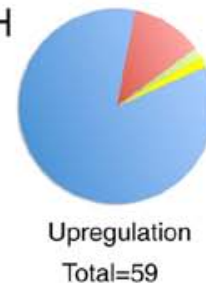

E
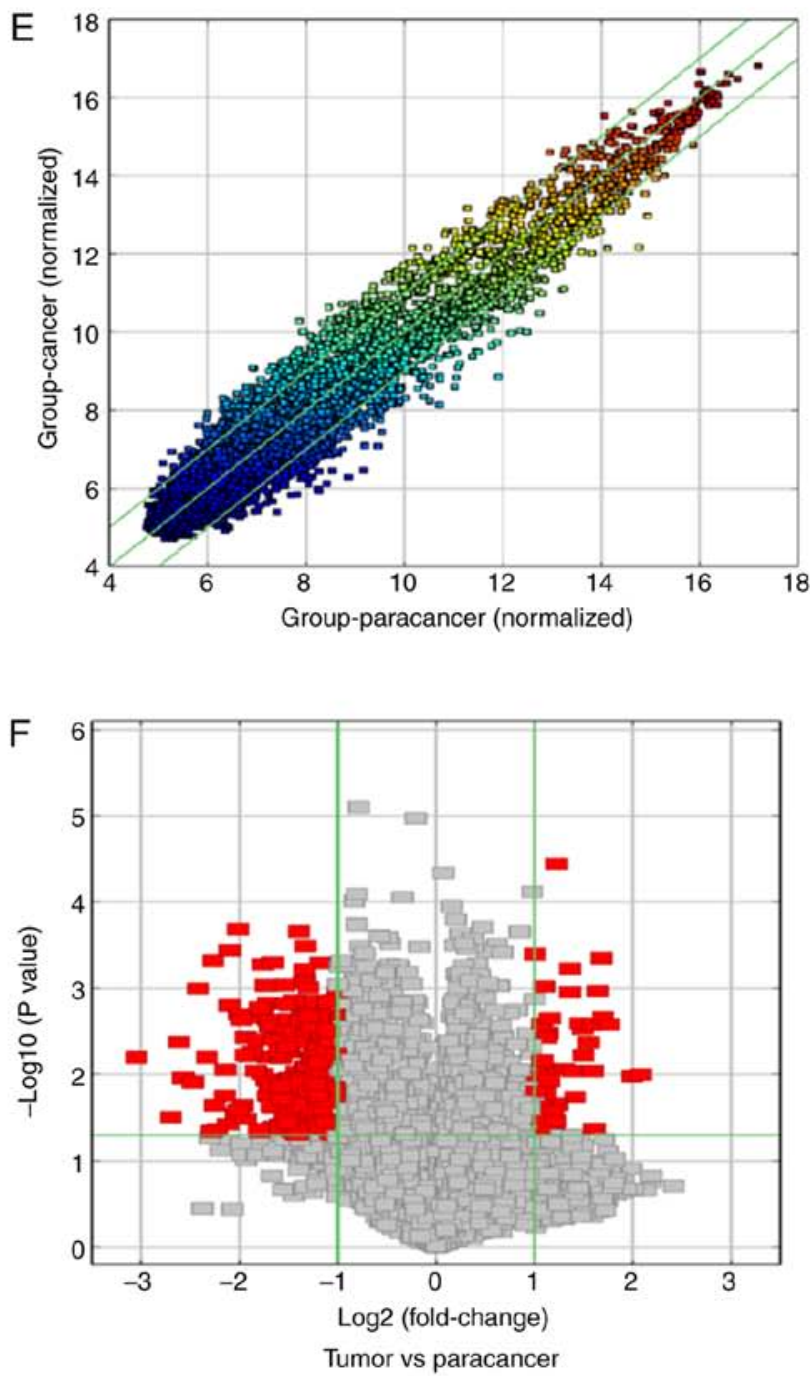

Figure 1. Analysis of differentially expressed circRNAs. Hematoxylin \& eosin staining for adjacent (A) normal tongue epithelium, (B) well-moderate and (C) poor-differentiated tumors. (D) Hierarchical clustering of the differentially expressed circRNAs. The color red indicates high relative expression, and green indicates low relative expression. (E) circRNAs in the scatter-plot above the top green line and below the bottom green line indicates $>2.0$-fold or $<0.5$-fold change of circRNAs between the adjacent normal and TSCC tissues. (F) circRNAs in the volcano plot represent the 2.0 -fold upregulated and downregulated circRNAs with statistical significance $(\mathrm{P}<0.05)$. (G) Summary of microarray results; a total of 335 circRNAs were identified from the analysis. (H) Classification of dysregulated circRNAs displayed in pie charts. circRNAs, circular RNAs; TSCC, tongue squamous cell carcinoma. 


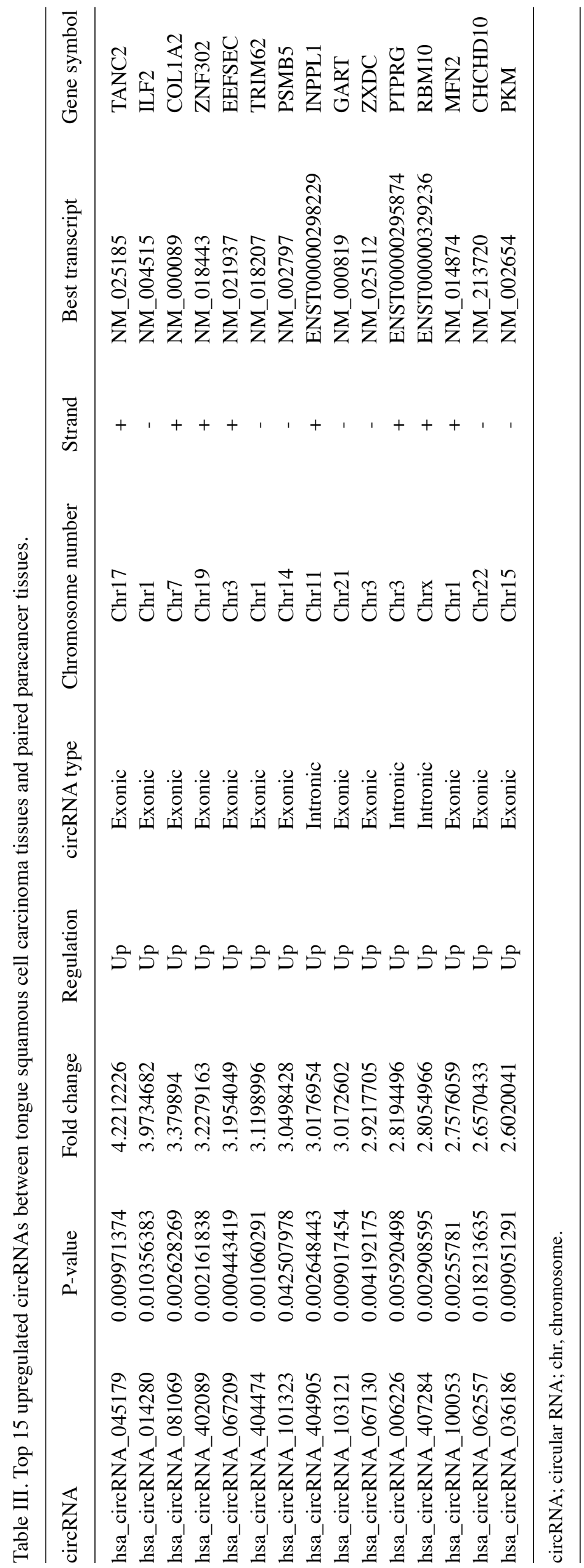




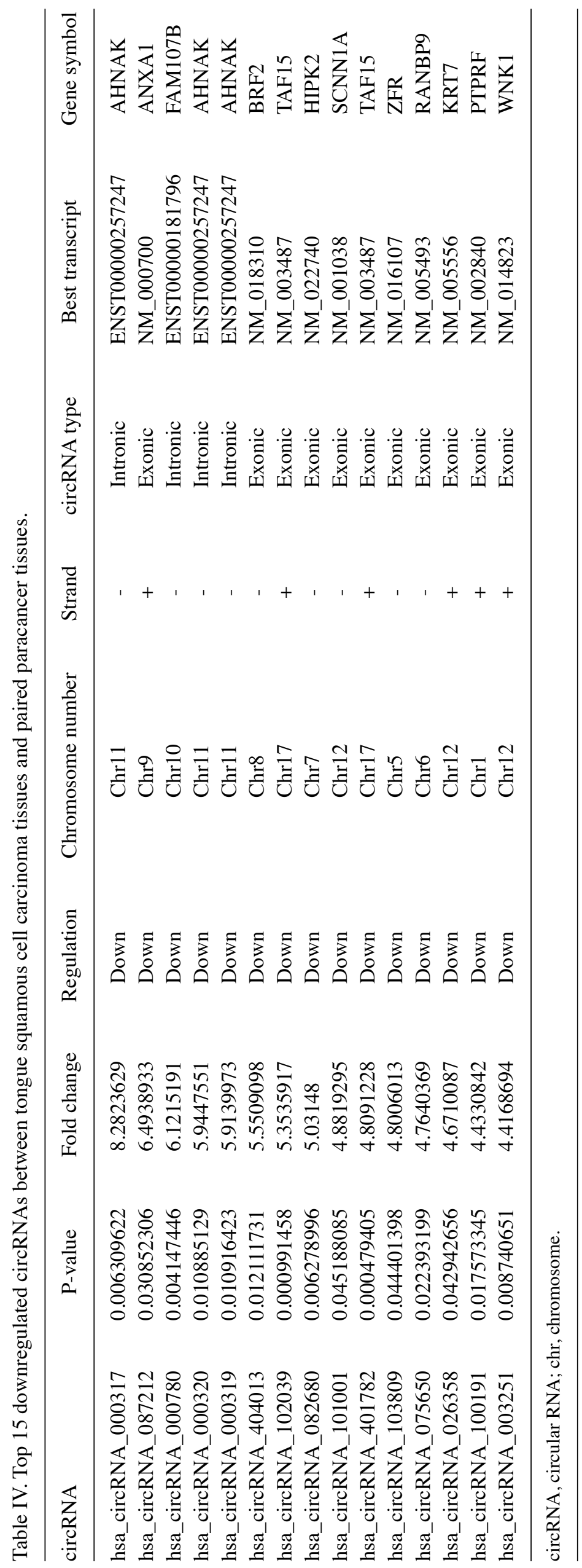




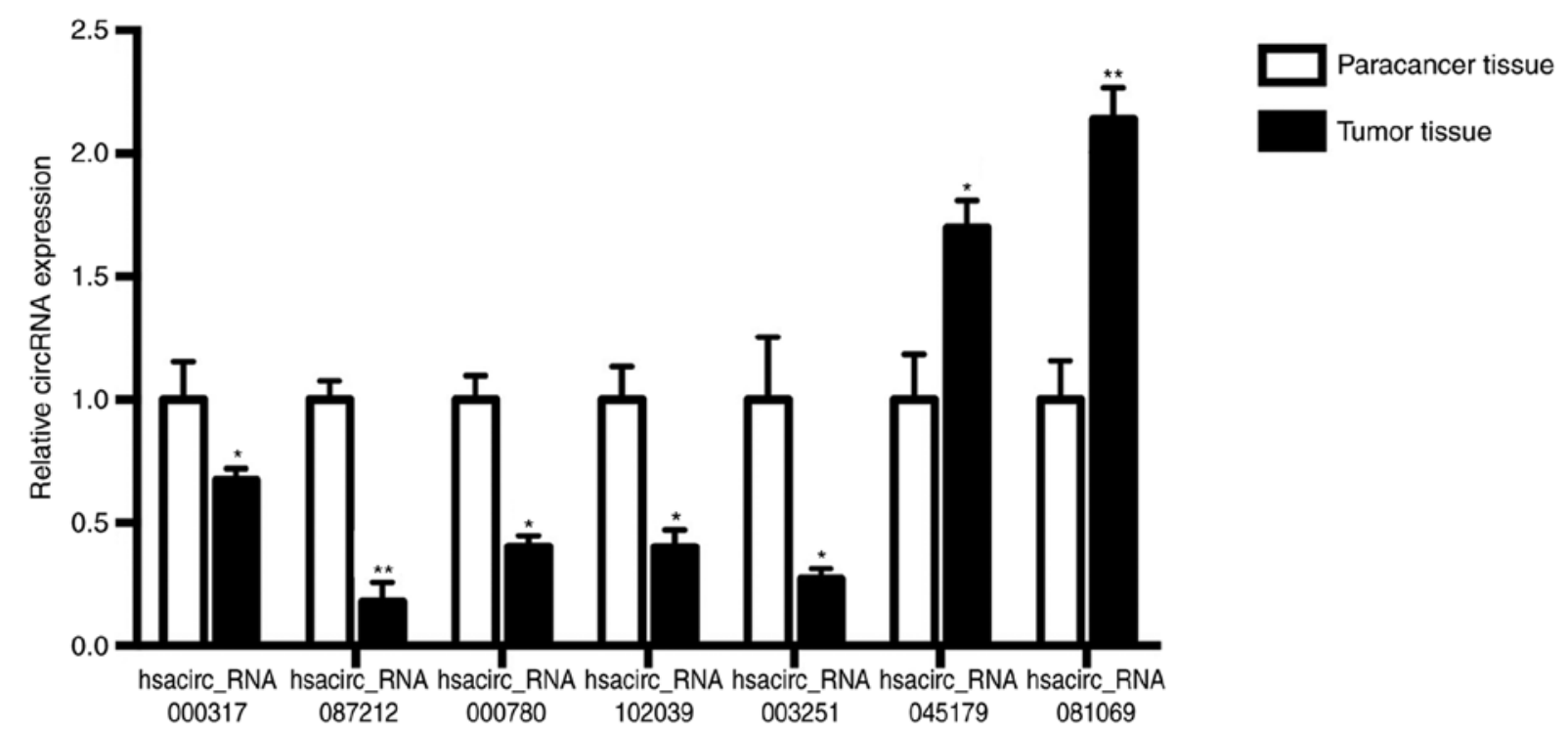

Figure 2. qPCR validation of selected differentially expressed circRNAs from microarray data. In total, seven circRNAs were selected for qPCR validation. Fold changes were calculated by the $2^{-\Delta \Delta C q}$ method. Data are representative of ten paired adjacent normal and TSCC tissues. Values are expressed as the mean \pm SEM. ${ }^{*} \mathrm{P}<0.05,{ }^{* * *} \mathrm{P}<0.01$ vs. paracancer tissue. circRNAs, circular RNAs; qPCR, quantitative PCR.

transcriptome profiles of TSCC and adjacent normal tissues. A total of 11,196 circRNAs were examined. After screening, 59 upregulated and 276 downregulated circRNAs were identified and their characteristics were analyzed. The results of the present study analyzed the circRNAs expression in TSCC, which may facilitate the complicated molecular mechanisms in TSCC development.

circRNAs represent a distinctive type of endogenous non-coding RNA. Due to their distinctive molecular structure, circRNAs have higher stability in different body fluids and exosomes compared with other types of RNAs, which makes them useful as novel diagnostic and prognostic biomarkers (16-18). circRNAs are categorized into three types: i) Exonic circRNAs; ii) intronic circRNAs; and iii) exon-intro circRNAs (EIciRNAs) (19). Exonic circRNAs consist of two or more exons, that function as microRNA sponges, and are mainly localized in cytoplasm (19). Intronic circRNAs and EIciRNAs are localized in the nucleus and function as transcriptional regulators of the parent gene $(20,21)$. CircRNAs can regulate gene expression at different stages, including transcription and translation (21). Previous studies have reported the important roles of circRNAs in cancer $(22,23)$. The altered expression of circRNAs has been confirmed to be associated with the malignant features of cancer cells, including their dysregulated proliferation, migration and invasion. circ_0001649 was found to be downregulated in several cancer types, including gastric cancer, hepatocellular cancer, and cholangiocarcinoma (22-24), and was found to act as a tumor suppressor and a diagnostic marker. circ_0008039 is upregulated in breast cancer, and can promote cell proliferation and migration by serving as a competing endogenous RNA of miR-432-5p (25). circHIPK3 was found to be significantly upregulated in hepatocellular cancer and bladder cancer, and its dysregulation affects the proliferation, migration and invasion of cancer cells by serving as a sponge for multiple miRNAs, including miR-124, miR-558 and miR-379 (26). circRNAs are also involved in the progression of oral cancer. Specifically, circ_100290 is upregulated in oral cancer tissues and functions as a competing endogenous RNA by sponging miR-29 family members, upregulating CDK6 expression, which accelerates cancer progression (27). There are still multiple circRNAs that remain to be investigated. Furthermore, the biogenesis, cellular locations, functions and molecular mechanisms of circRNAs require further investigation.

To the best of our knowledge, the expression profile of circRNAs in TSCC has not been previously investigated. In the present study, a number of dysregulated circRNAs were identified. Among these circRNAs, circRNA_081069 and circRNA_087212 were the most significantly upregulated and downregulated, respectively. To the best of our knowledge, there is no detailed research of the function and underlying mechanisms of these circRNAs. In the present study, it was found that knockdown of circ_081069 suppressed the migratory and proliferative ability of TSCC cells, suggesting that this circRNA may have an oncogenic effect. Since circRNAs can act as miRNA sponges, bioinformatic analysis was conducted to predict the possible interacting miRNAs, and it was predicted that circ_081069 was potentially able to interact with miR-665. miR-665 was found to suppress osteosarcoma cell proliferation and invasion by inhibiting the small $\mathrm{G}$ protein Rab23 (14). In the present study, the potential interaction between circ_081069 and miR-665 was investigated, and luciferase activity assay showed that miR-665 directly targeted circ_081069. In addition, miR-29a was predicted to interact with circ_087212. miR-29a was identified to regulate malignant cell features and drug resistance in several cancer types, including hepatocellular cancer and lung cancer by targeting Sirtuin 1 and NRAS $(15,28)$. Based on the present results, identifying novel approaches that may target and affect the stability of the interaction between circRNA and miRNAs may be important for inhibiting cancer metastasis, invasiveness and recurrence. 
A hsa_miR-4733-3p vs hsa_circRNA_081069
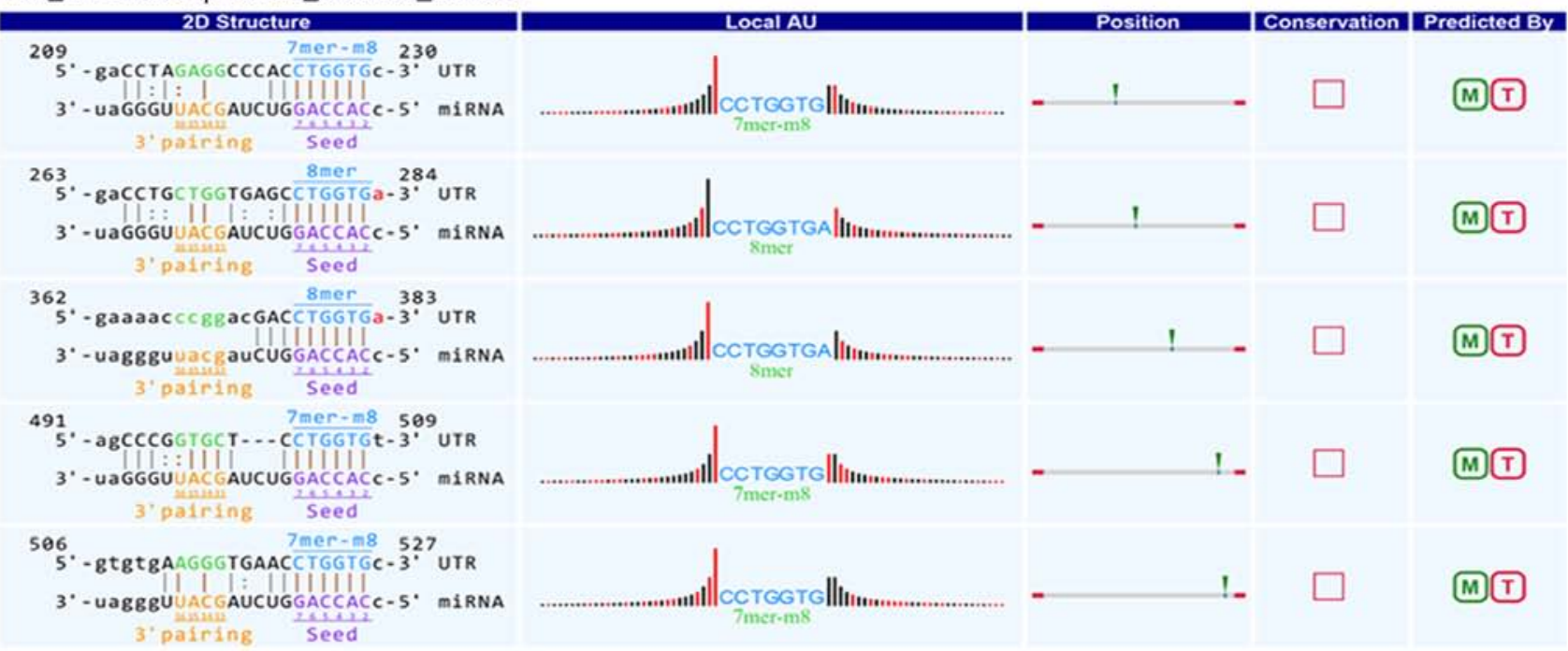

hsa_miR-665 vs hsa_circRNA_081069

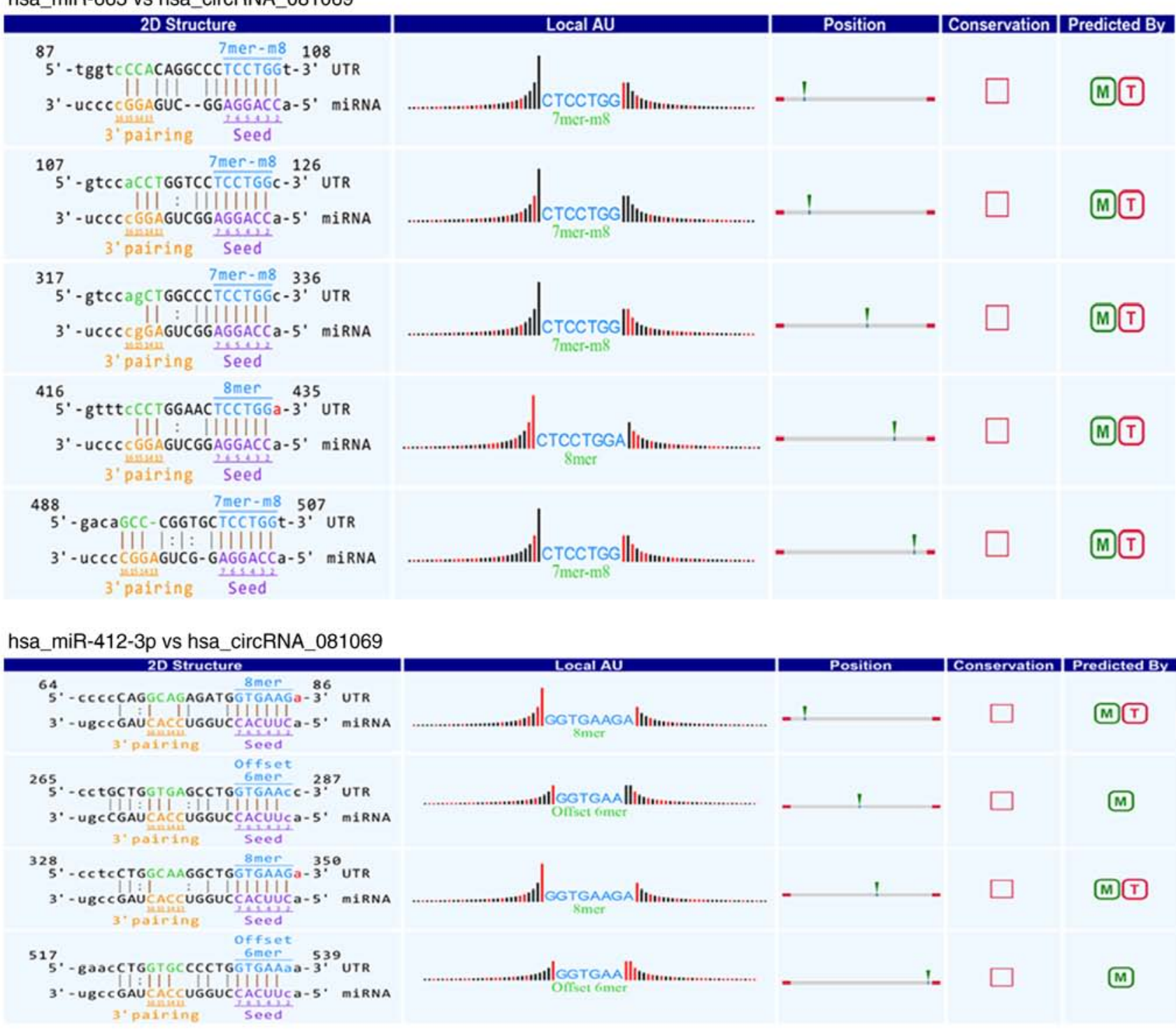

Figure 3. Detailed annotation for circRNA/miRNA interactions. (A) circRNA_081069.

However, additional studies are required to further clarify the circRNA-miRNA network in TSCC.
The present study identified circRNAs profiles in TSCC and the possible circRNA-miRNA interactions. 
B hsa_miR-29a-5p vs hsa_circRNA_087212

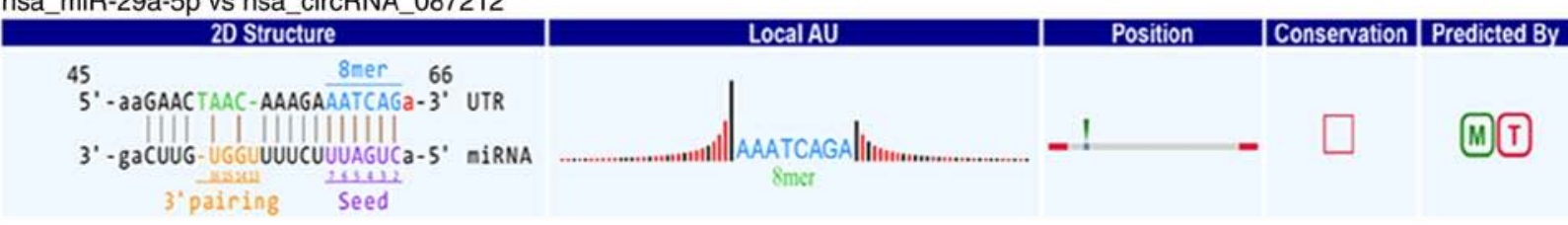

hsa_miR-6873-3p vs hsa_circRNA_087212

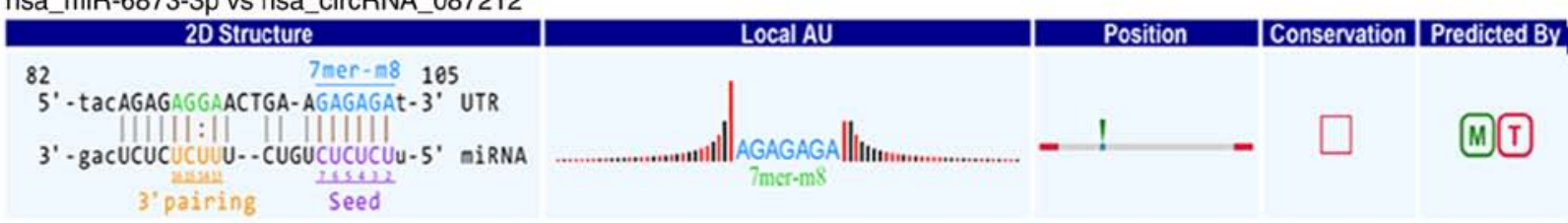

hsa_miR-6809-3p vs hsa_circRNA_087212

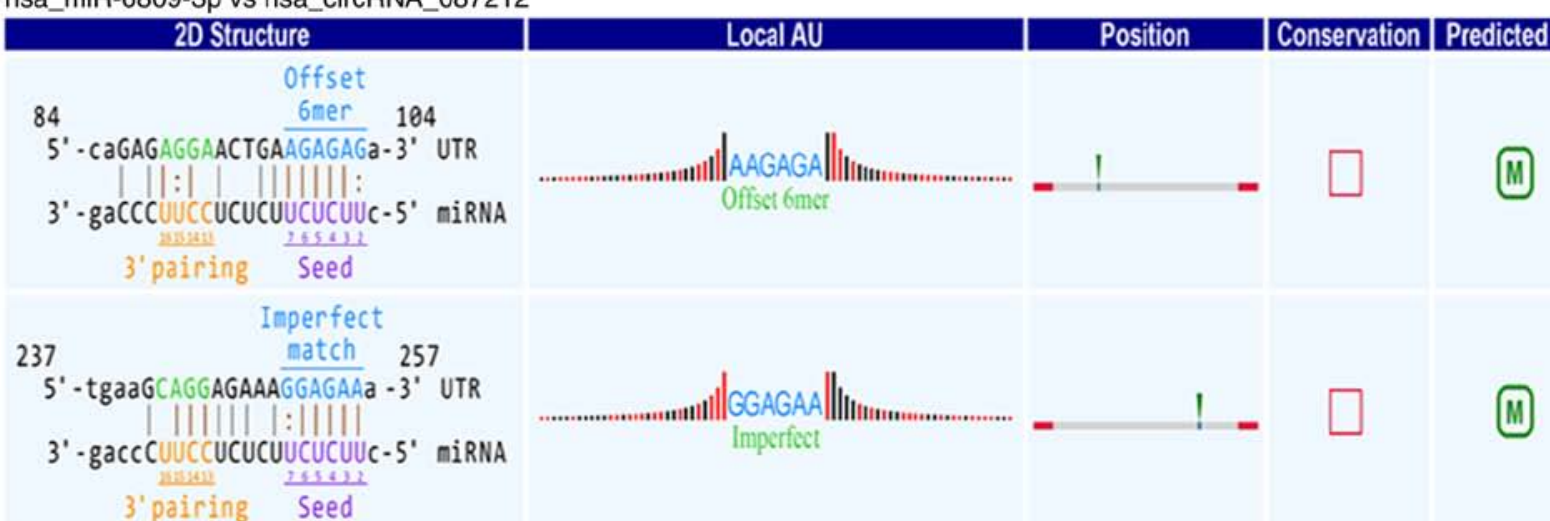

hsa_miR-6515-3p vs hsa_circRNA_087212

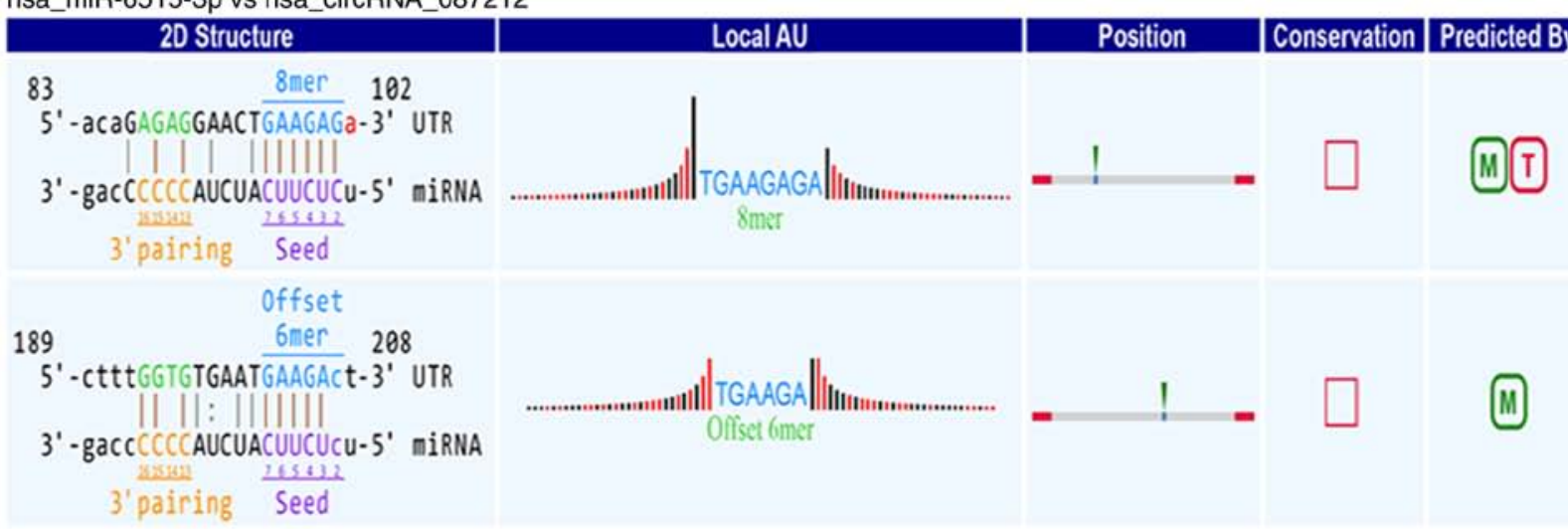

hsa_miR-5584-3p vs hsa_circRNA_087212

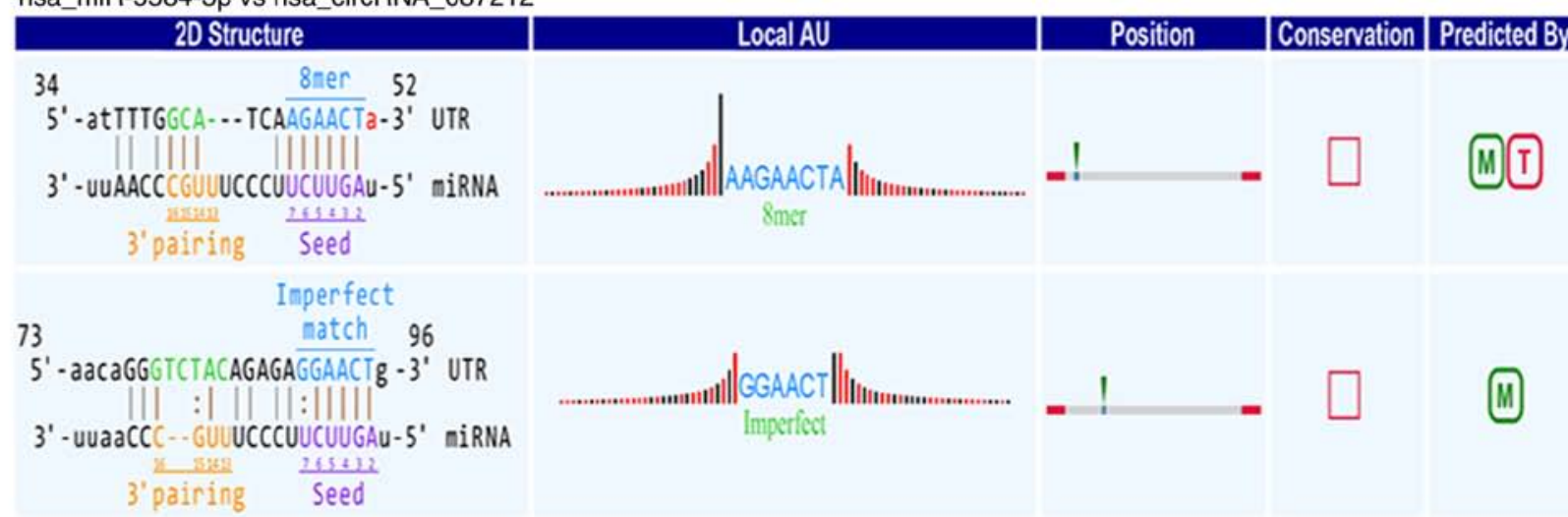

Figure 3. Continued. Detailed annotation for circRNA/miRNA interactions. (B) circRNA_087212. '8mer' indicates that the bases from number two to eight match perfectly, and the number one base is A. '7mer-m8' indicates that the bases from number two to eight match perfectly, and the number one base is not A. '6mer' indicates that the bases from number two to seven match perfectly, and the number one base is not A. 'offset 6mer' indicates that the bases from number three to eight match perfectly. 'Imperfect match' indicates that there is an imperfect base match from number two to seven. M, circRNA/miRNA interaction could be predicted by miRanda. T, circRNA/miRNA interaction could be predicted by TargetScan. circRNAs, circular RNAs; miRNA, microRNA; A, adenosine. 

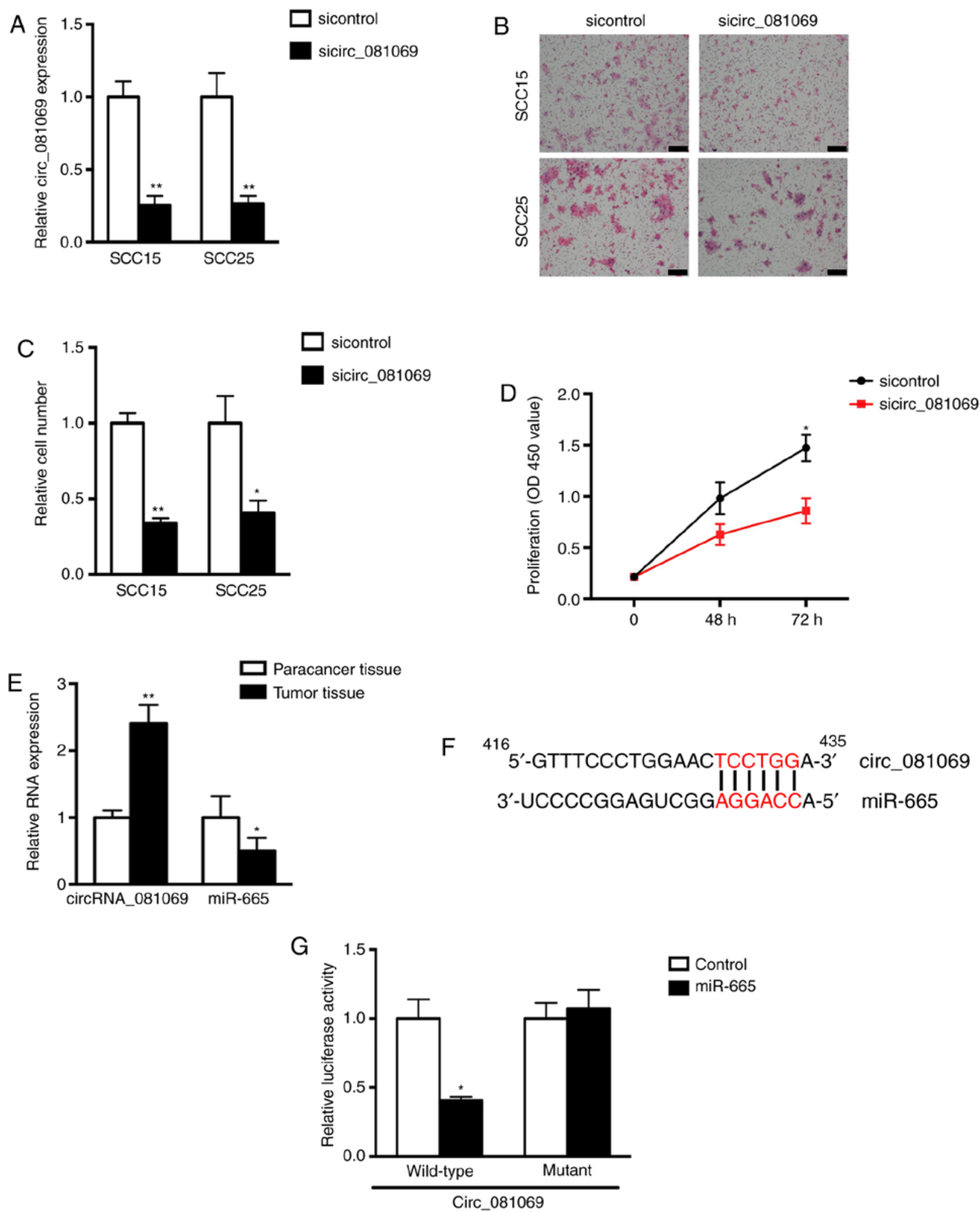

Figure 4. miR-665 interacts with circ_081069. (A) circ_081069 expression was decreased after siRNA transfection in SCC15 and SCC25 cell lines. (B) Representative images of cell migration at $24 \mathrm{~h}$. Scale bars, $200 \mu \mathrm{m}$. (C) circ_081069 knockdown inhibited the migratory ability of SCC15 and SCC25 cells. (D) circ_081069 knockdown inhibited the proliferative ability of SCC15 cells. (E) miR-665 is downregulated in TSCC tissues from the four tested patients. (F) Binding sites between circ_081069 and miR-665 predicted by bioinformatic analysis. (G) Luciferase reporter assay showed that miR-665 overexpression inhibited the activity of the wild-type circ_081069 reporter but not that of the mutant reporter. Values are presented as the mean \pm SEM from three independent experiments. ${ }^{*} \mathrm{P}<0.05,{ }^{* *} \mathrm{P}<0.01$ vs. corresponding control. circRNAs, circular RNAs; miRNA, microRNA; siRNA, silencing RNA; sicirc_081069, siRNA targeting circ_081069.

The present findings may increase the understanding of TSCC carcinogenesis and development, facilitating the development of novel approaches for the diagnosis and treatment of TSCC.

\section{Acknowledgements}

The authors would like to thank Dr Xin Cong (Department of Physiology and Pathophysiology, Peking University 
Health Science Center and Key Laboratory of Molecular Cardiovascular Sciences) for her kind suggestions.

\section{Funding}

The present work was supported by The Peking University School of Stomatology Postdoctoral Fund (grant no. YS0203), The Research Foundation of Peking University School and Hospital of Stomatology (grant no. PKUSS20190102) and The Doctoral Start Up Fund of Beijing Hospital (grant no. bj-2018-022).

\section{Availability of data and materials}

The datasets used and/or analyzed during the current study are available from the corresponding author on reasonable request.

\section{Authors' contributions}

TW performed the cell experiment and wrote the manuscript. PY collected the tissue samples and analyzed the data. GYY designed the study. ZYZ designed the experimental study and revised the manuscript. All the authors read and approved the final manuscript.

\section{Ethics approval and consent to participate}

The study was approved by The Ethics Committee of Peking University School and Hospital of Stomatology (approval no. PKUSSIRB-2013009). The patient consents were acquired before tissue collection.

\section{Patient consent for publication}

Not applicable.

\section{Competing interests}

The authors declare that they have no competing interests.

\section{References}

1. Patel RS, Clark JR, Dirven R, Wyten R, Gao K and O'Brien CJ: Prognostic factors in the surgical treatment of patients with oral carcinoma. ANZ J Surg 79: 19-22, 2009.

2. Yuen PW, Lam KY, Chan AC, Wei WI and Lam LK: Clinicopathological analysis of local spread of carcinoma of the tongue. Am J Surg 175: 242-244, 1998.

3. Rodriguez T, Altieri A, Chatenoud L, Gallus S, Bosetti C, Negri E, Franceschi S, Levi F, Talamini R and Lavecchia C: Risk factors for oral and pharyngeal cancer in young adults. Oral Oncol 40: 207-213, 2004.

4. Memczak S, Jens M, Elefsinioti A, Torti F, Krueger J, Rybak A, Maier L, Mackowiak SD, Gregersen LH, Munschauer M, et al: Circular RNAs are a large class of animal RNAs with regulatory potency. Nature 495: 333-338, 2013.

5. Jeck WR, Sorrentino JA, Wang K, Slevin MK, Burd CE, Liu J, Marzluff WF and Sharpless NE: Circular RNAs are abundant, conserved, and associated with ALU repeats. RNA 19: 141-157, 2013.

6. Ebbesen KK, Hansen TB and Kjems J: Insights into circular RNA biology. RNA Biol 14: 1035-1045, 2017.

7. Wang Y, Mo Y, Gong Z, Yang X, Yang M, Zhang S, Xiong F, Xiang B, Zhou M, Liao Q, et al: Circular RNAs in human cancer. Mol Cancer 16: 25, 2017.
8. Hansen TB, Kjems J and Damgaard CK: Circular RNA and miR-7 in cancer. Cancer Res 73: 5609-5612, 2013.

9. Dong R, Zhang XO, Zhang Y, Ma XK, Chen LL and Yang L: CircRNA-derived pseudogenes. Cell Res 26: 747-750, 2016.

10. Brierley JD, Gospodarowicz MK and Wittekind C (eds): TNM Classification of Malignant Tumours, 8th edition. Wiley, Chichester, 2017.

11. Wu HJ, Zhang CY, Zhang S, Chang M and Wang HY: Microarray expression profile of circular RNAs in heart tissue of mice with myocardial infarction-induced heart failure. Cell Physiol Biochem 39: 205-216, 2016

12. Livak KJ and Schmittgen TD: Analysis of relative gene expression data using real-time quantitative PCR and the 2(-Delta Delta C(T)) method. Methods 25: 402-408, 2001.

13. Guo XH, Wang JY, Gao Y, Gao M, Yu GY, Xiang RL, Li L, Yang NY, Cong X, Xu XY, et al: Decreased adiponectin level is associated with aggressive phenotype of tongue squamous cell carcinoma. Cancer Sci 104: 206-213, 2013.

14. Dong C, Du Q, Wang Z, Wang Y, Wu S and Wang A: MicroRNA- 665 suppressed the invasion and metastasis of osteosarcoma by directly inhibiting RAB23. Am J Transl Res 8: 4975-4981, 2016.

15. Zhang Y, Yang L, Wang S, Liu Z and Xiu M: MiR-29a suppresses cell proliferation by targeting SIRT1 in hepatocellular carcinoma. Cancer Biomark 22: 151-159, 2018.

16. Li Y, Zheng Q, Bao C, Li S, Guo W, Zhao J, Chen D, Gu J, He X and Huang S: Circular RNA is enriched and stable in exosomes: A promising biomarker for cancer diagnosis. Cell Res 25: 981-984, 2015.

17. Dou Y, Cha DJ, Franklin JL, Higginbotham JN, Jeppesen DK, Weaver AM, Prasad N, Levy S, Coffey RJ, Patton JG and Zhang B: Circular RNAs are down-regulated in KRAS mutant colon cancer cells and can be transferred to exosomes. Sci Rep 6: 37982, 2016.

18. Lasda E and Parker R: Circular RNAs co-precipitate with extracellular vesicles: A possible mechanism for circRNA clearance. PLoS One 11: e0148407, 2016.

19. Zhang XO, Wang HB, Zhang Y, Lu X, Chen LL and Yang L: Complementary sequence-mediated exon circularization. Cell 159: 134-147, 2014

20. Zhang Y, Zhang XO, Chen T, Xiang JF, Yin QF, Xing YH, Zhu S, Yang $L$ and Chen LL: Circular intronic long noncoding RNAs. Mol Cell 51: 792-806, 2013.

21. Li Z, Huang C, Bao C, Chen L, Lin M, Wang X, Zhong G, Yu B, $\mathrm{Hu}$ W, Dai L, et al: Exon-intron circular RNAs regulate transcription in the nucleus. Nat Struct Mol Biol 22: 256-264, 2015.

22. Qin M, Liu G, Huo X, Tao X, Sun X, Ge Z, Yang J, Fan J, Liu L and Qin W: Has circ 0001649: A circular RNA and potential novel biomarker for hepatocellular carcinoma. Cancer Biomark 16: 161-169, 2016.

23. Xu Y, Yao Y, Zhong X, Leng K, Qin W, Qu L, Cui Y and Jiang X: Downregulated circular RNA has circ 0001649 regulates proliferation, migration and invasion in cholangiocarcinoma cells. Biochem Biophys Res Commun 496: 455-461, 2018.

24. Li WH, Song YC, Zhang H, Zhou ZJ, Xie X, Zeng QN, Guo K, Wang T, Xia P and Chang DM: Decreased expression of has circ 00001649 in gastric cancer and its clinical significance. Dis Markers 2017: 4587698, 2017.

25. Liu Y, Lu C, Zhou Y, Zhang Z and Sun L: Circular RNA has circ 0008039 promotes breast cancer cell proliferation and migration by regulating miR-432-5p/E2F3 axis. Biochem Biophys Res Commun 3: 358-363, 2018.

26. Zheng Q, Bao C, Guo W, Li S, Chen J, Chen B, Luo Y, Lyu D, Li Y, Shi G, et al: Circular RNA profiling reveals an abundant circHIPK 3 that regulates cell growth by sponging multiple miRNAs. Nat Commun 7: 11215, 2016.

27. Chen L, Zhang S, Wu J, Cui J, Zhong L, Zeng L and Ge S: CircRNA 100290 plays a role in oral cancer by functioning as a sponge of the miR-29 family. Oncogene 36: 4551-4561, 2017.

28. Liu X, Lv X, Yang Q, Jin H, Zhou W and Fan Q: MicroRNA-29a functions as a tumor suppressor and increases cisplatin sensitivity by targeting NRAS in lung cancer. Technol Cancer Res Treat 17: 1533033818758905, 2018.

This work is licensed under a Creative Commons Attribution-NonCommercial-NoDerivatives 4.0 International (CC BY-NC-ND 4.0) License. 